4aPP9. Source spectrum recovery at different spatial locations. Ewan A. Macpherson (Waisman Ctr., Univ. of Wisconsin-Madison, 1500 Highland Ave., Madison, WI 53705-2280)

The directional filtering of sound by the pinnae is vital to localization, but distorts the spectrum of the signal reaching the eardrum. Sounds do not appear to change character dramatically as a function of direction, so listeners might have some ability to deconvolve pinna effects from the received signal. A three-interval profile analysis task measured listeners' ability to recover source spectra. The stimuli were wideband noise bursts in which the levels of $1 / 3$-octave bands were adjusted to control spectral shape. They were filtered by listeners' measured head-related transfer functions (HRTFs) and delivered via headphones. Listeners discriminated between various nonflat spectra and perturbed versions of them. HRTFs were selected randomly for each interval, and localizability was controlled by varying the correspondence of the left and right HRTFs. Thresholds were highest when HRTFs were imposed diotically, and lowest in an unfiltered diotic baseline condition. Accurate spatialization improved recovery, but applying independent near-ear HRTFs dichotically was more effective. However, deconvolution was imperfect in both conditions. The results suggest that the ability to disregard HRTF filtering and achieve some timbre constancy over direction depends on having two independent, wideband "looks" at the source spectrum, rather than on accurate localization.

4aPP10. The role of temporal factors in pitch perception. Valter Ciocca (Dept. of Speech \& Hearing Sciences, Univ. of Hong Kong, 34 Hospital Rd., Hong Kong)

This study investigated how pitch perception mechanisms integrate acoustic information over time. The pitch matching procedure developed by Moore et al. [J. Acoust. Soc. Am. 77, 1853-1860 (1985)] was used in order to measure pitch shifts in a harmonic series (target complex) produced by mistuning a harmonic that either preceded or followed the target complex. In the first experiment, the mistuned component could either stop as the target complex started (pretarget condition) or start as the target stopped (post-target condition). The results showed that pitch shifts were significantly larger in the post-target than in the pretarget condition. In the second experiment, the duration of the silent period, which separated the mistuned component and the target complex, was varied in both the preand the post-target conditions. Pitch shifts were virtually eliminated by a delay longer than $20 \mathrm{~ms}$ in the pretarget condition. By contrast, a delay of $160 \mathrm{~ms}$ was necessary to eliminate pitch shifts in the post-target condition. These results suggest that pitch perception mechanisms take into account the order of occurrence of acoustic information for calculating the pitch of a complex sound. [Work supported by Hong Kong RGC, Grant HKU 362/94M.]

4aPP11. Glide difference limens as a function of center frequency, duration, and transition size. John P. Madden (Dept. of Commun. Disord., Univ. of North Dakota, University Station, Grand Forks, ND 58202-8040)

The study determined glide difference limens for up and down glides in several conditions. Signal durations were 50,100 , and $400 \mathrm{~ms}$, and the average stimulus center frequencies were 2 and $6 \mathrm{kHz}$. The standard, or comparison, signals changed in frequency by 0,500 , and $1000 \mathrm{~Hz}$. These transition sizes were chosen to cover the range of $F 2$ formant transitions. The subject's task was to distinguish between the standard and a target signal with a greater or lesser change in frequency, depending on the experimental condition, in a 2-alternative, forced-choice task. To avoid the confounding effect of between-stimuli static pitch cues, the center frequencies of the standard and target stimuli were "roved" about the center frequencies [Neill and Feth, J. Acoust. Soc. Am. Suppl. 1 87, S23 (1990)]: The data were analyzed using a level-detection model consisting of a filter bank, a nonlinearity, a temporal integrator, and a detection device. The question of whether the results support a rate place or temporal mechanism of frequency coding is discussed. [Work supported by NIDCD.]

\title{
Session 4aSA
}

\section{Structural Acoustics and Vibration: Statistical Methods in Complex Structures I}

\author{
Richard L. Weaver, Chair \\ Department of Theoretical and Applied Mechanics, University of Illinois, 104 Wright Street, Urbana, Illinois 61801
}

Invited Papers

4aSA1. Parameter estimation for fuzzy structures. A result concerning vibrations in the low-frequency range. Christian Soize (Structures Dept., ONERA, BP 72, 92322 Chatillon Cedex, France)

In the field of structural vibrations, the structural complexity can be induced by "secondary" mechanical subsystems attached to the "master" structure or by "local eigenmodes" of some continuum elastic subelements of the master structure; these local eigenmodes induce a structural complexity when the model of these subelements can only restitute the elastostatic behavior but not its elastodynamic response. Within this context, a model is presented of the apparent vibration damping of the master structure due to the vibrations of the structural complexity. This vibration-damping model is deduced from the theory of fuzzy structures that was previously developed by the author. Presently, this model uses only the mean part of the probabilistic fuzzy law of the fuzzy substructure. A model of the generalized damping matrix deduced from the model of the structural complexity, is explicitly constructed. This generalized damping matrix depends on parameters related to the fuzzy substructure. Problems related to the model parameters estimation are studied. Finally, an example is presented and allows the theory to be validated.

\section{9:00}

4aSA2. Fuzzy elements, their coupling rules, and the Jaynes-Shannon maximum entropy principle. Allan D. Pierce (Boston Univ., Dept. of Aerospace and Mech. Eng., 110 Cummington St., Boston, MA 02215)

Emerging theories of fuzzy structures are regarded as the wholesale replacement of certain portions of the structure by fuzzy elements, whose chief characteristic is a smeared-out (fuzzied) distribution of natural frequencies, so that there are an infinite number of natural frequencies in any given frequency band. Descriptors of fuzzy elements are the mass per unit natural frequency band and 